\title{
SIMULACION NUMERICA DEL ESFUERZO DE FLEXION A CARGAS VARIABLES EN ENGRANAJES PLASTICOS
}

\section{NUMERICAL SIMULATION OF THE BENDING STRESSES TO VARYING LOADS IN PLASTIC GEARS}

\author{
Jorge Laureano Moya Rodríguez ${ }^{1}$, Tito Roberto Vílchez Vílchez ${ }^{2}$
}

\begin{abstract}
RESUMEN
Los ensayos en bancos de prueba permiten simular las condiciones del contacto real que se generan entre los dientes de un engranaje, pero son costosos y de limitada utilidad por su precisión que depende de las condiciones del equipo y el material. El objetivo fundamental de esta investigación es desarrollar un procedimiento que simule el esfuerzo de flexión en dientes de engranajes de plástico a través del método de simulación numérica y realizar el estudio de los principales parámetros que afectan el desempeño de los engranajes plásticos como son: torque, potencia y esfuerzos de estos engranajes cuando se encuentren en régimen de operación, haciendo una comparación con los aceros fundidos. Los resultados de esta investigación satisfacen los objetivos generales y específicos del estudio, los que contribuirán en la mejora de la tecnología de la industria peruana.
\end{abstract}

Palabras clave.- Engranajes, Simulación numérica, Cálculo analítico, MEF, Flexión.

\begin{abstract}
The tests on test rigs to simulate the real contact conditions generated between the teeth of a gear, but are expensive and of limited utility for its accuracy depends on the condition of equipment and material. The objective of this research is to develop a procedure that simulates the bending stress in gear teeth plastic through numerical simulation method and the study of the main parameters that affect the performance of plastic gears such as torque, power and efforts of these gears when in operation regime, comparing with the molten steel. The results of this research meet the general and specific objectives of the study, which will help in improving the technology of Peruvian industry.
\end{abstract}

Keywords.- Gears, Numerical simulation, Analytical calculation, MEF, Bending.

\section{INTRODUCCIÓN}

Las construcciones mediante el uso de plástico están presentes en todos los ámbitos de la sociedad. Los avances conseguidos por esta en los últimos 30 años no habrían sido posibles sin este material que es fundamental en sectores como la agricultura, la industria, la alimentación, la medicina, las telecomunicaciones y el transporte. En los últimos años el uso de materiales plásticos en la Industria Mecánica ha ido proliferando para convertirse en un material de uso primordial, especialmente en la fabricación de engranajes, reduciendo así los costos, el peso, el ruido, etc. Por otra parte los engranajes plásticos pueden usarse en medios donde no pueden trabajar los engranajes metálicos contribuyendo de esta forma como un factor fundamental para la protección del medio ambiente. Los ensayos son una parte muy importante dentro del desarrollo de cualquier

\footnotetext{
${ }^{2}$ Dr. Vice Decano de Investigaciones y Posgrado Facultad de Mecánica Universidad Central Marta Abreu de las Villas Cuba, ${ }^{1}$ M.Sc Ing. Docente investigador de la Facultad de Ingeniería Mecánica de la Universidad Nacional de Ingeniería.
} 
producto y vital en el caso de fabricación de engranajes, dado que permiten verificar que el piñón o engranaje no tendrán ningún problema debido al diseño pronosticado para el periodo de su vida útil. La planificación de los ensayos debe determinar qué tipos de ensayos hay que realizar.

En consecuencia, los centros con bancos de pruebas son instalaciones muy importantes dentro de los centros tecnológicos.

Según la experiencia de los científicos internacionales, acerca de que, ningún componente de una transmisión por engranajes plásticos diseñado, si no se mide, es imposible predecir acertadamente su capacidad de torque, tersura, ruido, rendimiento y la expectativa de su vida útil. Para ello es necesaria la investigación actualizada de una transmisión ensamblada y el uso de un banco de pruebas. Los engranajes plásticos son fabricados en la actualidad por varios procedimientos entre los que se encuentran los métodos tradicionales por arranque de viruta y el moldeo, $[1,2,3,4]$.

La inyección de plástico es una forma que adquiere cada vez más auge en la fabricación de estas transmisiones por las ventajas que reporta desde el punto de vista constructivo y de calidad de la pieza obtenida.

Profundas diferencias existen entre el engranaje moldeado y los engranajes elaborados por arranque de viruta tanto en aceros como en plásticos, en el último método según la calidad de la superficie a elaborar depende del régimen de corte a que estará sometida, pues, se corre el riesgo de que la herramienta utilizada no copie exactamente el perfil deseado, influyendo negativamente en la vida de este.

Ahora bien, al analizar un engranaje moldeado se pueden señalar varias ventajas que van desde la calidad en la superficie obtenida hasta una buena concentricidad entre sus parámetros geométricos donde se logra además una alta precisión, los que se verifican a través de un banco de pruebas.

Las precisiones en los engranajes plásticos están estrechamente ligadas a los métodos de fabricación utilizados en el molde y en particular en el macho y en la cavidad. Al elaborar el macho y la cavidad del molde deben tenerse en cuenta regímenes tecnológicos que garanticen el acabado superficial óptimo en correspondencia con las características exigidas a la pieza plástica así como del material a moldear, además de facilitar el trabajo del molde en su conjunto $[5,6,7,8,9,10,11,12,13$ y 14$]$.

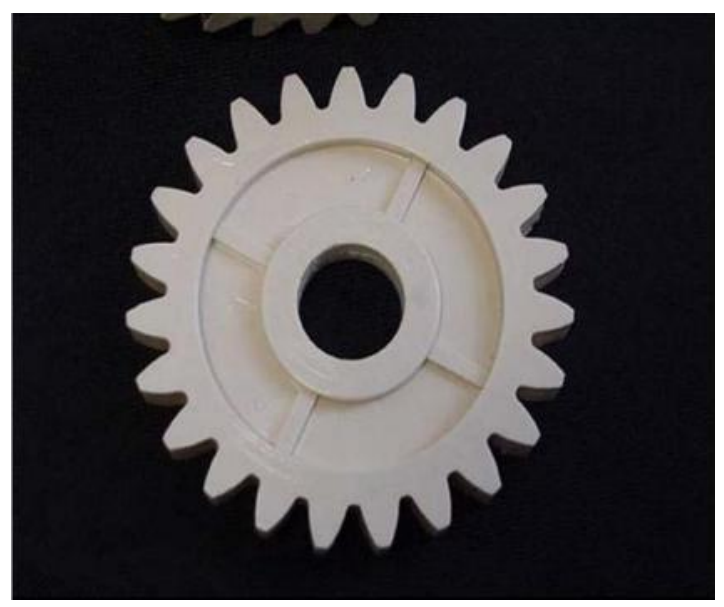

Fig. 1 Vista frontal de un engranaje fabricado donde se aprecia la geometría del diente.

\section{FACTIBILIDAD DE CONSTRUCCION DE ENGRANAJES PLASTICOS EN EL PERU}

En nuestro país es necesario realizar el protocolo sobre el empleo de los engranajes plásticos con posibilidades de elaborarlos con dientes simétricos y asimétricos, con el propósito fundamental de lograr engranajes plásticos más resistentes que los normales y con bajos costos en la fabricación de los mismos, considerando que en el Perú no existen Bancos de Ensayo de Engranajes.

Resulta interesante exponer que en el análisis del estado del arte se pueden constatar propiedades de los plásticos que los hacen ventajosos en algunas aplicaciones.

Hay criterios recogidos en diferentes fuentes internacionales ya citadas anteriormente como son:

- Eliminar o reducir el consumo de lubricantes.

- Facilidad en maquinar o moldear.

- Posibilidad del uso del código de colores, lo que reduce errores de ensamblaje.

- Son más económicos.

Como un aspecto esencial, se han propuesto sustituciones de ruedas metálicas por plásticas, sin 
destacar la sustitución de ruedas plásticas de dientes normales por las de dientes asimétricos, con lo que también se logran resultados destacados.

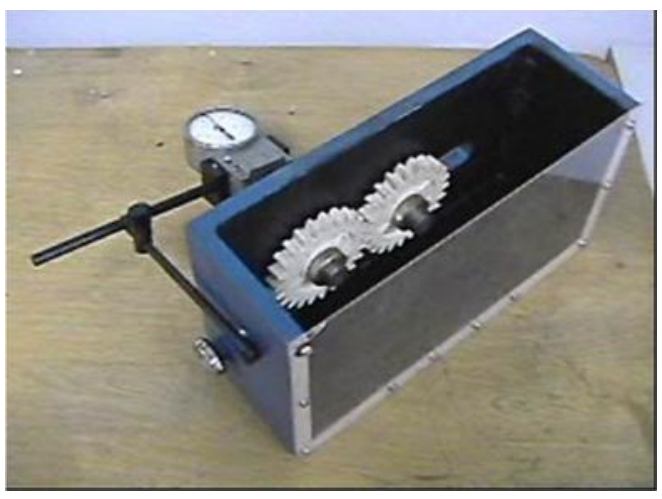

Fig. 2 Vista superior de un banco de pruebas con su instrumentación.

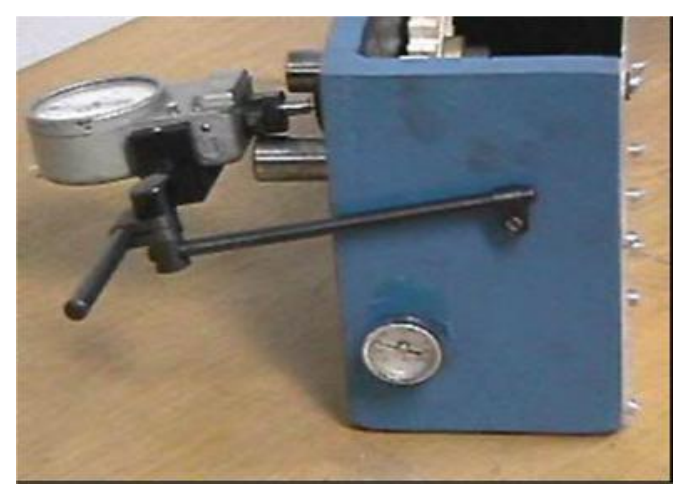

Fig. 3 Engranajes fabricados con el molde.

\section{CALCULO DE ESFUERZOS EN ENGRANAJES PLASTICOS MEDIANTE METODOS TEORICOS}

Para la realización de las comparaciones pertinentes.

Los materiales ensayados son Nylon, Acetal, Torlon y Acero fundido, como decisión de diseño se considera el número de dientes del piñon $\mathrm{Zp}=$ 25 dientes, del engranaje $\mathrm{Zg}=100$ dientes para todos los casos, con un coeficiente de asimetría $\mathrm{C}$ $=1 \mathrm{y}$ la distancia entre centros $\mathrm{d}_{\mathrm{C}}=250 \mathrm{~mm}$. Variando las potencias de entrada con $3 \mathrm{~kW}, 4 \mathrm{~kW}$ $\mathrm{y}$ con $5 \mathrm{~kW}$.

De los resultados obtenidos, sobre la influencia que tiene la variación de potencia en el funcionamiento de la transmisión de los engranajes plásticos, en primer lugar contrastaremos los resultados de los esfuerzos en la base del diente para cada material plástico según el FEM y los métodos experimentales de Dvorak, Kelley y Faires que es el método teórico más real en comparación con los métodos teóricos conocidos de otros autores.

Para una frecuencia angular $\mathrm{N}=900 \mathrm{RPM}$ se determina la rapidez del diente $\mathrm{V}=305,36 \mathrm{~m} / \mathrm{min}$ para una fuerza tangencial de $F_{t}=982 \mathrm{~N}$. Según el método de Faires:

$F_{d}=\frac{(200+3.28 V)}{(200+0.82 V)} F_{t}=\frac{(200+3.28(305,36))}{(200+0.82(305,36))}(982)=2619,8 \mathrm{~N}$

$F_{d}=F_{S}=\frac{S \cdot b \cdot m \cdot Y}{10}$

$\sigma=\frac{10(267)}{(2.54)(4)(0.57)}\left(\frac{(9,8)\left(10^{4}\right)}{10^{6}}\right)\left(\frac{0,9}{2}\right)=20,3 M P a$

$\mathrm{F}_{\mathrm{d}}=267 \mathrm{~kg}$

Asimismo, utilizando el método de Dvorak, tenemos: $\mathrm{V}=305,36 \mathrm{~m} / \mathrm{min}$

$S=\frac{55(6+V)}{F Y V} \cdot(W) \cdot P \cdot C_{S}$

$\sigma=\frac{55(6+1011,42)(6,7) \cdot(6,35)(1)}{(1)(0.424)(1011,42)}\left(\frac{6.84949}{1000}\right)\left(\frac{0,9}{2}\right)=17,1 \mathrm{MPa}$

De igual forma utilizando el método de Kelley,

tenemos:

$$
\begin{aligned}
& S=\frac{W_{t} \cdot P}{F Y} \\
& \sigma=\frac{(242,4489) \cdot(6,35)}{(1)(0,424)}\left(\frac{6.84949}{1000}\right)\left(\frac{0,9}{2}\right)=11,1 M P a
\end{aligned}
$$




\section{ANALISIS MEDIANTE SIMULACION PARA LA EVALUACION DE ENGRANAJES FERROSOS Y PLASTICOS}

\section{Uso del método de elementos finitos MEF}

El método de los elementos finitos como formulación matemática es relativamente nuevo; aunque su estructura básica es conocida desde hace bastante tiempo, en los últimos años ha sufrido un gran desarrollo debido a los avances informáticos. Han sido precisamente estos avances informáticos los que han puesto a disposición de los usuarios gran cantidad de programas que permiten realizar cálculos con elementos finitos. El manejo correcto de este tipo de programas exige un profundo conocimiento no solo del material con el que se trabaja, sino también de los principios del MEF. Sólo en este caso estaremos en condiciones de garantizar que los resultados obtenidos en los análisis se ajustan a la realidad.

Actualmente el método se encuentra en una fase de gran expansión: es ampliamente utilizado en la industria y continúan apareciendo cientos de trabajos de investigación en este campo. Los ordenadores han aportado el medio eficaz de resolver la multitud de ecuaciones que se plantean en el MEF, cuyo desarrollo práctico ha ido caminando parejo de las innovaciones obtenidas en el campo de la arquitectura de los ordenadores. Entre éstas, además de permitir la descentralización de los programas de $\mathrm{EF}$, ha contribuido a favorecer su uso a través de sofisticados paquetes gráficos que facilitan el modelado y la síntesis de resultados.

Hoy en día ya se concibe la conexión inteligente entre las técnicas de análisis estructural, las técnicas de diseño (CAD), y las técnicas de fabricación. El método de elementos finitos (MEF) utiliza la generación de mallas y la geometría de la malla es uno de los factores que influyen en la precisión del resultado. Los últimos avances en este campo indican que el futuro tecnológico está en métodos de adaptación de orden superior, que responde satisfactoriamente a la creciente complejidad de las simulaciones de ingeniería y satisface la tendencia general la resolución simultánea de los fenómenos con múltiples escalas. Entre las diversas estrategias de adaptación para los elementos finitos, los mejores resultados se pueden lograr con la-adaptabilidad con el objetivo de mejorar la resolución en una cantidad específica. Existen ejemplos donde la 'adaptabilidad' resulta ser la única manera de resolver el problema en un nivel requerido de exactitud.

\section{Limitaciones}

En general el MEF tal como se usa actualmente tiene algunas limitaciones: El MEF calcula soluciones numéricas concretas y adaptadas a unos datos particulares de entrada, no puede hacerse un análisis de sensibilidad sencillo que permita conocer como variará la solución si alguno de los parámetros se altera ligeramente. Es decir, proporciona sólo respuestas numéricas cuantitativas concretas, no relaciones cualitativas generales.

El MEF proporciona una solución aproximada cuyo margen de error en general es desconocido. Si bien algunos tipos de problemas permiten acotar el error de la solución, debido a los diversos tipos de aproximaciones que usa el método, los problemas no-lineales o dependientes del tiempo en general no permiten conocer el error. En el MEF la mayoría de aplicaciones prácticas requiere mucho tiempo para ajustar detalles de la geometría, existiendo frecuentemente problemas de mal condicionamiento de las mallas, desigual grado de convergencia de la solución aproximada hacia la solución exacta en diferentes puntos, etc. En general una simulación requiere el uso de numerosas pruebas y ensayos con geometrías simplificadas o casos menos generales que el que finalmente pretende simularse, antes de empezar a lograr resultados satisfactorios.

En función de la naturaleza de las variables de diseño se pueden definir dos tipos de análisis de sensibilidad:

Sensibilidad de parámetros.- Se definen como parámetros aquellas variables de diseño que aparecen explicitas en la formulación del problema dinámico, por ejemplo el modulo elástico del piñón.

Sensibilidad de formas.- En este otro caso, las variables de diseño están relacionadas con las dimensiones básicas del problema o con la posición de los dientes como elementos. Por ejemplo, al tomar como variables de diseño las coordenadas de las conexiones nodales en la base 
del diente. El correcto análisis de sensibilidad está ligado con la adecuada parametrización de cada elemento. La capacidad de transmisión de potencia es la capacidad cuando la carga tangencial causa que el esfuerzo esperado sea igual al número del esfuerzo admisible, si se consideran todos los factores de modificación. En estas condiciones el piñón es el más crítico respecto del esfuerzo flexionante. Con la finalidad de buscar un balance comparativo entre los materiales ferrosos versus los materiales plásticos, que cada día van mejorando su resistencia para una misma condición de trabajo.

Se analizó el acero y plásticos para determinar su valor de esfuerzo máximo dentro del límite elástico (Sy) en el pie del diente mediante el método de elementos finitos (MEF) con el software ANSYS 14.0 con un mallado de más de 76.000 nodos y 50.000 elementos sólidos tetraedros de alto orden para la realización de las comparaciones pertinentes, utilizando un elemento tridimensional hexa10 (tetraedro de 10 nodos).

Los materiales simulados son Nylon, Torlon y acero fundido, como decisión de diseño se considera el número de dientes del piñón $\mathrm{Zp}=25$ dientes, del engrane $\mathrm{Zg}=100$ dientes para todos los casos, el coeficiente de asimetría $\mathrm{C}=1 \mathrm{y}$ la distancia entre centros $d_{C}=250 \mathrm{~mm}$.

Asimismo para engranajes para potencias medias o bajas en engranajes plásticos se utiliza una eficiencia del $90 \%$. Variando las potencias de entrada con $3 \mathrm{~kW}, 4 \mathrm{~kW}$ y con $5 \mathrm{~kW}$ y con los parámetros que se muestra, como datos iniciales se considera una transmisión por engranajes con las siguientes características:

Altura de la cabeza del diente:

$8.64 \mathrm{~mm}$

Ancho del diente:

Velocidad angular del Piñon:

Coeficiente de huelgo radial:

Modulo:

Angulo de la herramienta:

$25,4 \mathrm{~mm}$

900 RPM

0.16

$4 \mathrm{~mm}$

$20^{\circ}$

\section{Simulación con acero}

El acero $\left(\mathrm{E}=1.9 \times 10^{11} \mathrm{MPa}\right)$ es recomendable para la construcción de engranajes, se muestra los resultados del cálculo de esfuerzos de este material a través del MEF:

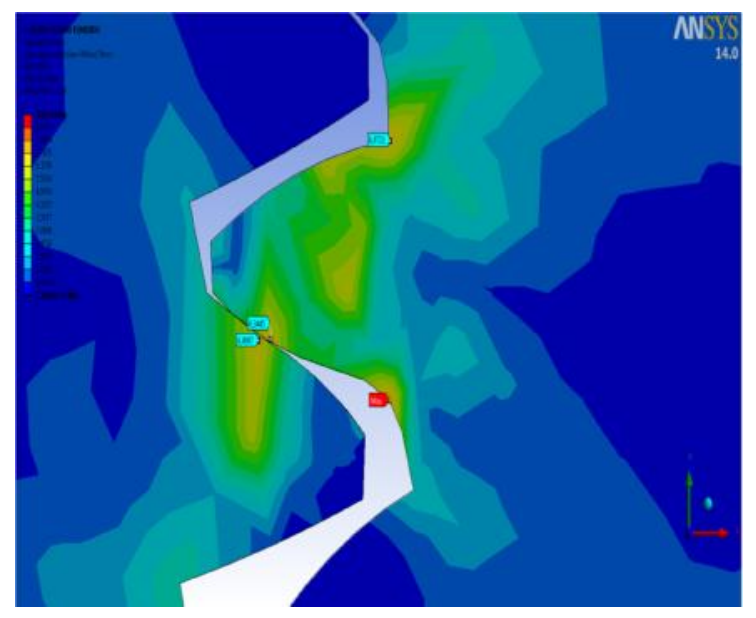

Fig. 4 Esfuerzos en el pie del diente del acero.

Tabla 1. Valores de esfuerzos en el pie del diente del acero según la potencia transmitida.

\begin{tabular}{cc}
\hline Potencia $(\mathbf{k W})$ & Esfuerzo (MPa) \\
\hline 3 & 8.6514 \\
4 & 12.945 \\
5 & 17.239 \\
\hline
\end{tabular}

\section{Simulación con nylon}

El nylon es una designación genérica para una familia de polímeros sintéticos conocidos como poliamidas, primero producidos el 28 de febrero de 1935, por Wallace Carothers en la Estación experimental de Du Pont en la ciudad de Wilmington, USA. 


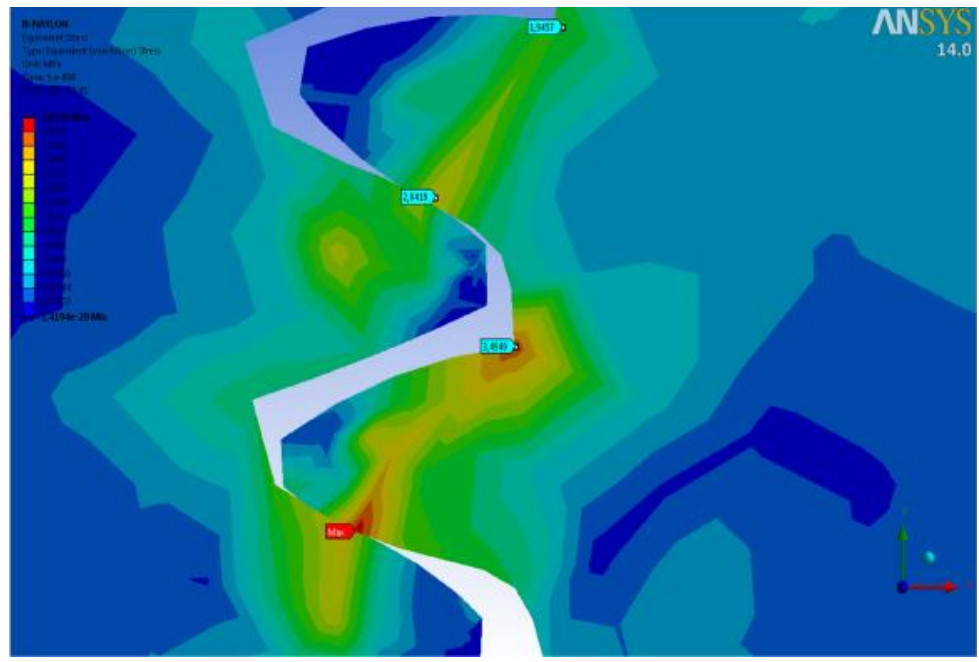

Fig. 5 Esfuerzos en el pie del diente del nylon.

Tabla 2. Valores de esfuerzos en el pie del diente del Nylon según la potencia transmitida.

\begin{tabular}{cc}
\hline POTENCIA $(\mathbf{k W})$ & ESFUERZO (MPa) \\
\hline 3 & 3.8741 \\
4 & 5.0424 \\
5 & 6.191 \\
\hline
\end{tabular}

\section{Simulación con acetal}

El acetal o poli-oxi-metileno, es también un termoplástico de mejores prestaciones que la poliamida. Este tiene alta resistencia a la compresión, bajos coeficientes de rozamiento y excepcional aguante de los impactos. Sus características principales son: Alta resistencia mecánica, rigidez y dureza, muelle elástico muy elevado, alta resistencia a los choques, también a temperaturas bajo cero, muy buena estabilidad dimensional, buenas propiedades de deslizamiento y gran facilidad de mecanización.

El acetal absorbe muy poca agua, por lo tanto, a la abrasión debido al rozamiento se desgasta con mucha más facilidad si es de acetal que si es de nylon o poliéster, y en consecuencia la vida de la pieza es menor.

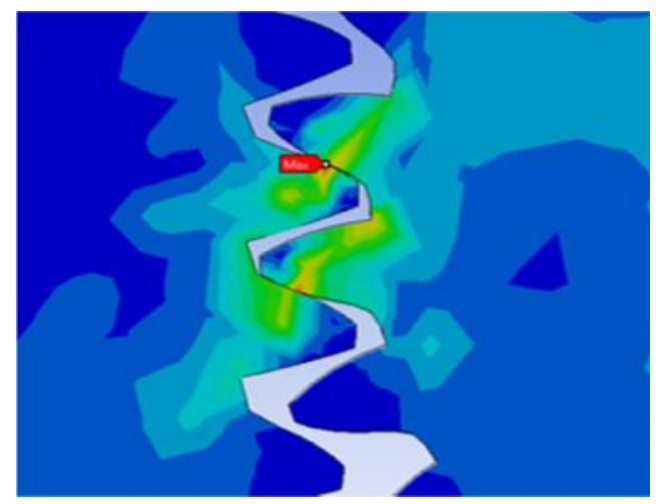

Fig. 6 Esfuerzos en el pie del diente del acetal.

Tabla 3. Valores de esfuerzos en el pie del diente del Acetal según la potencia transmitida. 


\begin{tabular}{cc}
\hline Potencia $(\mathbf{k W})$ & Esfuerzo (MPa) \\
\hline 3 & 5.4189 \\
4 & 7.4976 \\
5 & 9.5754 \\
\hline
\end{tabular}

\section{Simulación con torlon}

El torlon de poliamida-imida (PAI) es uno de los últimos plásticos de alta resistencia con la mayor resistencia y rigidez de cualquier termoplástico hasta $275^{\circ} \mathrm{C}\left(525^{\circ} \mathrm{F}\right)$.

Tiene una excelente resistencia al desgaste, fluencia y a los productos químicos incluyendo ácidos fuertes y la mayoría de los compuestos orgánicos y es ideal para los entornos de servicio.

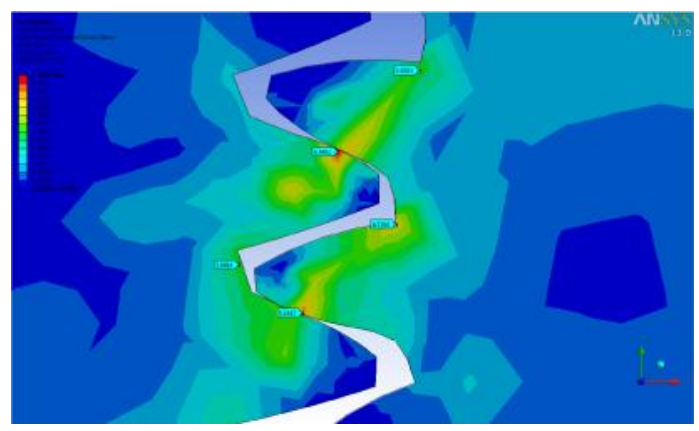

Fig. 7 Esfuerzos en el pie del diente del torlon.

Tabla 4. Valores de esfuerzos en el pie del diente del Torlon según la potencia transmitida.

\begin{tabular}{cc}
\hline POTENCIA $(\mathbf{k W})$ & ESFUERZO (MPa) \\
\hline 3 & 6.7352 \\
4 & 9.5181 \\
5 & 12.310 \\
\hline
\end{tabular}

Finalmente, en términos de resistencia, entre los materiales Nylon, Acetal y el Torlon, se determina a través de la simulación numérica, que el Torlon tiene mucha más resistencia que los otros materiales plásticos en las tres condiciones de trabajo a diferentes potencias, por cual se hace la comparación de su resistencia con la del acero:

Tabla 5. Valores de esfuerzos en el pie del diente de los materiales plásticos para una potencia de $5 \mathrm{~kW}$, con métodos experimentales y FEM.

MATERIAL POTENCIA DE TRABAJO: $5 \mathrm{~kW}$

ESFUERZO EN LA BASE DEL DIENTE (MPa)

FAIRES

DVORAK

KELLEY

MEF

$\begin{array}{lllll}\text { TORLON } & 20,3 & 17,1 & 11,16 & 12,31\end{array}$

Tabla 6. Valores de esfuerzos en el pie del diente de los materiales plásticos para una 
Potencia de $4 \mathrm{~kW}$, con métodos experimentales y FEM.

\begin{tabular}{ccccc}
\hline MATERIAL & \multicolumn{4}{l}{$\begin{array}{l}\text { POTENCIA DE TRABAJO: 4 kW } \\
\text { ESFUERZO EN LA BASE DEL DIENTE (MPa) }\end{array}$} \\
& FAIRES & DVORAK & KELLEY & MEF \\
\hline \multirow{2}{*}{ TORLON } & 16,2 & 13,6 & 8.13 & 9.5181 \\
\hline
\end{tabular}

Tabla 7. Valores de esfuerzos en el pie del diente de los materiales plásticos para una potencia de $3 \mathrm{~kW}$, con métodos experimentales y $\mathrm{MEF}$.

\begin{tabular}{|c|c|c|c|c|}
\hline \multirow[t]{2}{*}{ MATERIAL } & \multicolumn{4}{|c|}{$\begin{array}{l}\text { POTENCIA DE TRABAJO: } 3 \mathrm{~kW} \\
\text { ESFUERZO EN LA BASE DEL DIENTE (MPa) }\end{array}$} \\
\hline & FAIRES & DVORAK & KELLEY & MEF \\
\hline TORLON & 12,21 & 10,27 & 6,1 & 6,7352 \\
\hline
\end{tabular}

Como se puede observar, los métodos experimentales tradicionales considerando el tipo de material, enfocan con poca precisión en la determinación de la carga aplicada para determinar el esfuerzo en el pie del diente, sin embargo, con la utilización de los métodos computacionales como Cosmos Design Star, Nastran, Abaqus Finite Element y Ansys, el nivel de precisión es sumamente alto debido a la tecnología actual concentrando los nodos en las zonas de la base del diente, por lo cual, en todos los casos, los esfuerzos de trabajo son menores que los que corresponden a los métodos tradicionales.

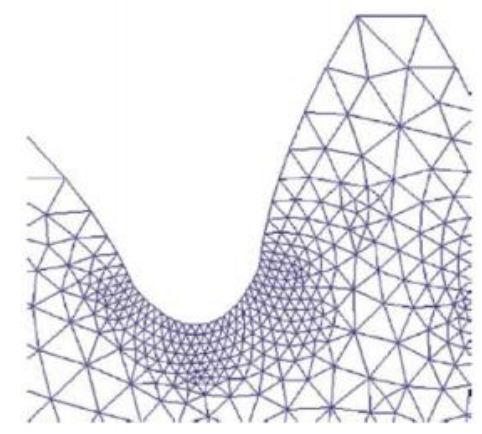

Fig. 8 Mallado con concentración de nodos en la base del diente del piñón. CONCLUSIONES
Actualmente existe una amplia gama de materiales plásticos que logran adaptarse a disímiles exigencias bajo las cuales, incluso, los metales trabajan deficientemente. El desarrollo alcanzado en la Industria del Plástico, unido a las bondades que este tiene desde el punto de sus propiedades mecánicas, su bajo peso e inercia, que se elimina o reduce el consumo de lubricantes y el nivel de ruido, su facilidad de maquinar o moldear, su compatibilidad con medios hostiles, la posibilidad del uso del código de colores y que son económicos, hacen que ganen cada día mayores aplicaciones en la técnica moderna y en particular en la construcción de transmisiones por engranajes, lo que aún no ha sido una realidad en Perú.

La síntesis de los engranajes plásticos difiere notablemente de la síntesis de los engranajes ferrosos. El modelo matemático por simulación desarrollado y sus expresiones permiten realizar la síntesis métrica de estos engranajes plásticos con gran precisión. Se pudo comprobar cómo la resistencia de los dientes de engranajes plásticos en el caso del Torlon es aproximadamente el $77 \%$ al que corresponde al acero aleado, bajo las mismas condiciones de carga a velocidades moderadas. La metodología del cálculo de los 
esfuerzos en el pie del diente de un engranaje plástico a través del FEM, resulta más preciso que las metodologías tradicionales.

Es factible la construcción de engranajes asimétricos corregidos usando las más modernas técnicas de diseño y fabricación. A través de sistemas CAD se obtiene los perfiles simétricos y asimétricos corregidos y exportando esta geometría a una electroerosiva de hilo, se logra fabricar la cavidad del molde y posteriormente con el molde diseñado y fabricado, se funden los engranajes plásticos.

\section{REFERENCIAS}

1. ANSI/AGMA 1006-A97. Annex F. "Generating Gear Geometry without Racks".

2. Broersma, I., "Design of gears". Editorial Industrial Press Inc., New York, 1975.

3. Ashley, S., "Plastic Gears saves pounds and parts". Revista Mechanical Engineering. May 1997, Vol 119, IIssue 5. ASME. USA.

4. B., Turčič., "Technological Analysis of Using Polymer Materials for Gears", report BMT 128/4/02, Prague, 15.9.2002.

5. Kelley, J. W., "Polymers get in gears". Revista Machine Design. Agosto 1996. Penton Publishing ISSN 0024-9114 USA.

6. "Diagnosing Problems with plastic gears". Revista Machine Design Julio 9, 1998. Penton Publishing ISSN 0024-9114 USA.

7. Fitzinger, D., "Basic Training for designing Plastic gears". Revista Machine Design. febrero 19, 1998. Penton Publishing ISSN 0024-9114 USA.

8. Fritzinger, D., "What to put on parts prints of plastic gears. Revista Machine Design". November 1998. Penton Publishing ISSN 0024-9114 USA.

9. García, A., "Diseño de Moldes de inyección para la fabricación de Engranajes Plásticos Asimétricos". Tesis presentada en Opción al Grado Académico de Master en Ciencias. Santa Clara. Cuba Noviembre del 2002.

10. Kleiss, R., Hoffmann, S., "The Generation of Precision Spurs Gears Through Wire Electrical Discharge Machining”. Technical Conference of the American Gear Manufacturer's Association, 1993.

11. Kleiss, R. E, Kapelevich, A. L., Kleiss, Jr. N. J., "New Opportunities with Molded gears", AGMA Fall Technical Meeting, Detroit, October 3-5, 2001, (01FTM9) Copyright - 2001 American Gear Manufacturers Association 1500 King Street, Suite 201 Alexandria, Virginia, 22314 October, 2001 ISBN: 1--55589--788-6.

12. Kleiss, R., Scott Hoffman, J., "The Generation of Precision Spur Gears Through Wire Electrical Discharge Machining". 1993 Technical Conference of the AGMA.

13. Kleiss, R., Must, E., "You Use Metal?". Revista Design Engineering. Marzo 2001.

Rogers Media, Publishing. One Mount Pleasant Road, 7th Floor. Toronto, Ontario. Canada M5W 1A7.

14. Kleiss, R., "The effect of thermal shrink and expansion on plastic gear geometry". 1993 AGMA Conference on Gearing.

Correspondencia: titovilchez@uni.edu.pe

Recepción de originales: noviembre 2012 Aceptación de originales: marzo 2013 